\title{
Quark Orbital Angular Momentum and Exclusive Processes at HERMES
}

\author{
FRANK ELLinghaUS \\ UNIVERSITY OF COLORADO \\ FOR THE HERMES-COLLABORATION \\ CiPAnP, Puerto Rico, May 2006
}

- Generalized Parton Distributions (GPDs)

- Exclusive Production of Photons (DVCS) And Mesons

- HERMES Results

- First model dependent constraint on Quark Orbital Angular Momentum 


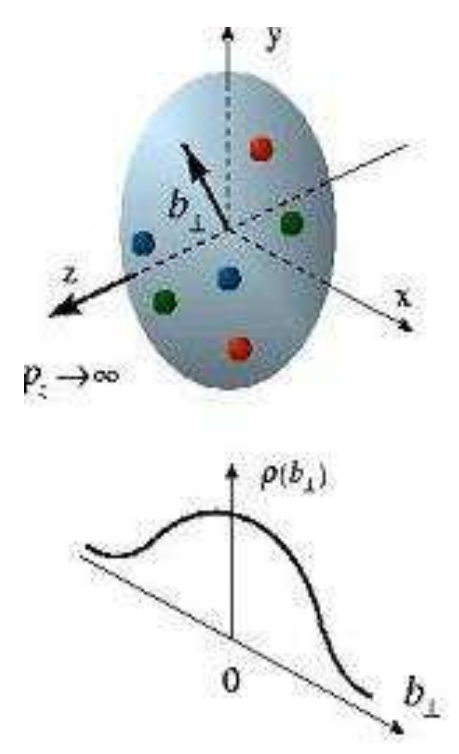

FF
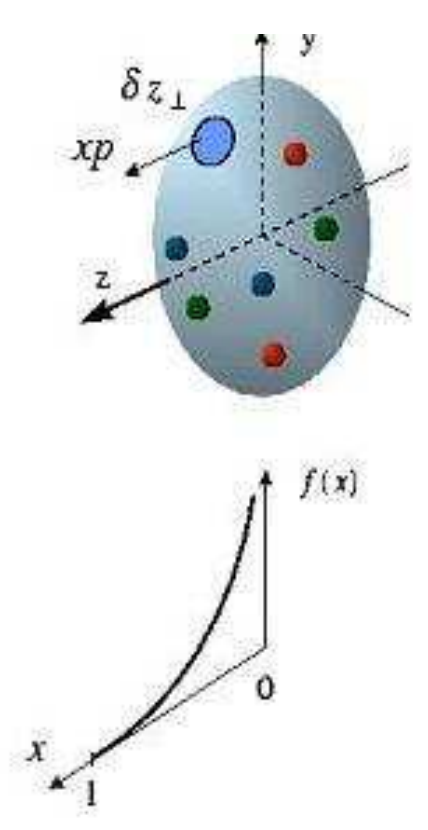

PDF

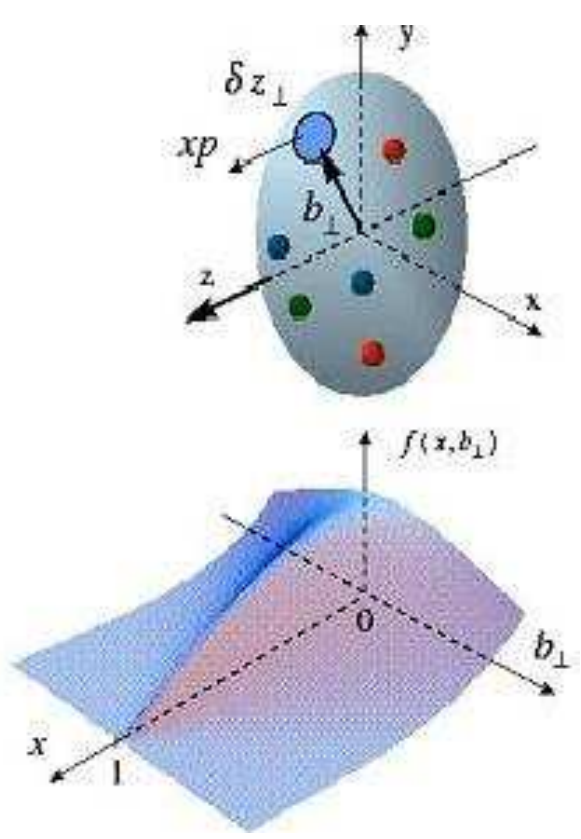

GPD

- Form Factors $\rightarrow$ Transverse position $\leftarrow$ Elastic sCattering

- PDFs $\rightarrow$ LOngitudinal MOMEntum Distribution $\leftarrow$ DiS

- GPDs $\rightarrow$ AcCess to transverse position And longitudinal MomenTUM Distr. AT THE SAME TIME, 3-D PICTURE $\leftarrow$ EXCLUSIVE REACTIONS 
Simplest/CLEANEST HARD EXCLUSIVE PROCESS:

DeEply-VirTual ELECTROPRoduction of REAL PHotons: $e p \rightarrow e^{\prime} p^{\prime} \gamma$

Deeply-virtual Compton Scattering (DVCS):

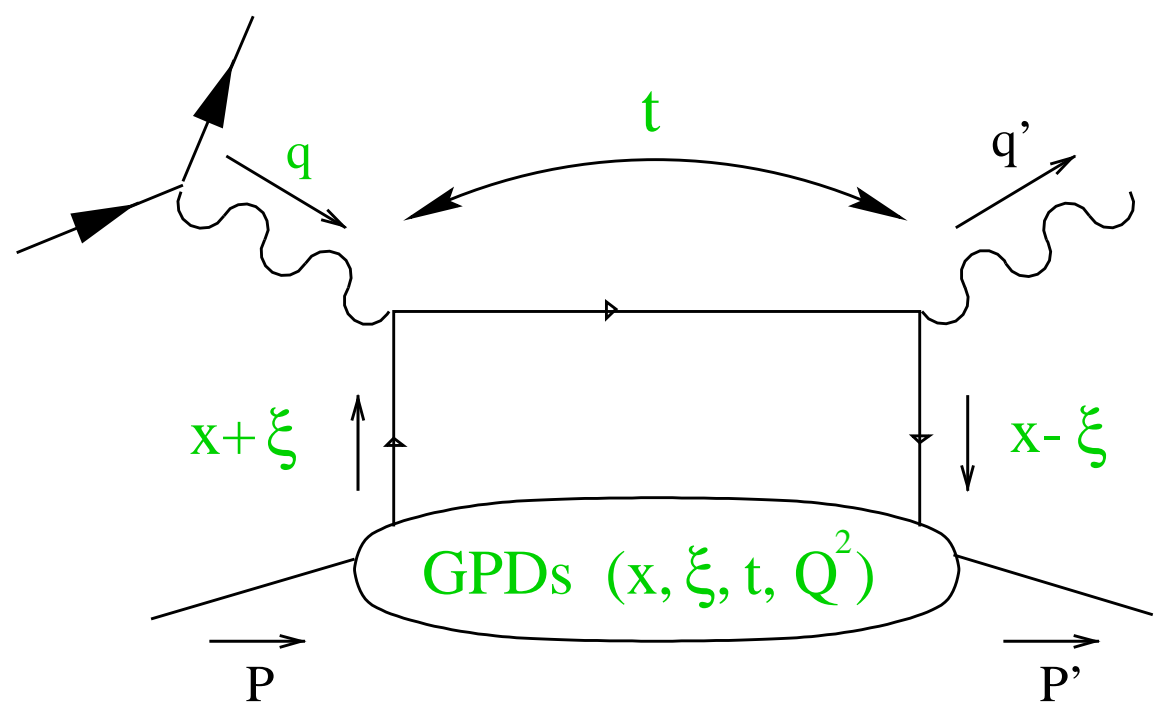

- Longitudinal momentum FRACTIONS:

$x \in[-1,1]$ (NOT ACCESSIBLE)

$\xi \approx x_{B} /\left(2-x_{B}\right)$

- $t=\left(q-q^{\prime}\right)^{2}$

$\left(\gamma^{*} \rightarrow \gamma\right.$ MOMENTUM TRANSFER $)$

- $Q^{2}=-q^{2}$

$\Rightarrow$ Measurements as FUnCtion of $x_{B}, t, Q^{2}$

DVCS: ACCESS TO ALL FOUR GPDs $H_{2} \widetilde{H}_{2} E, \widetilde{E}$

Mesons: Access to $H, E(\mathrm{VM})$ And $\widetilde{H}, \widetilde{E}(\mathrm{PS})$ 


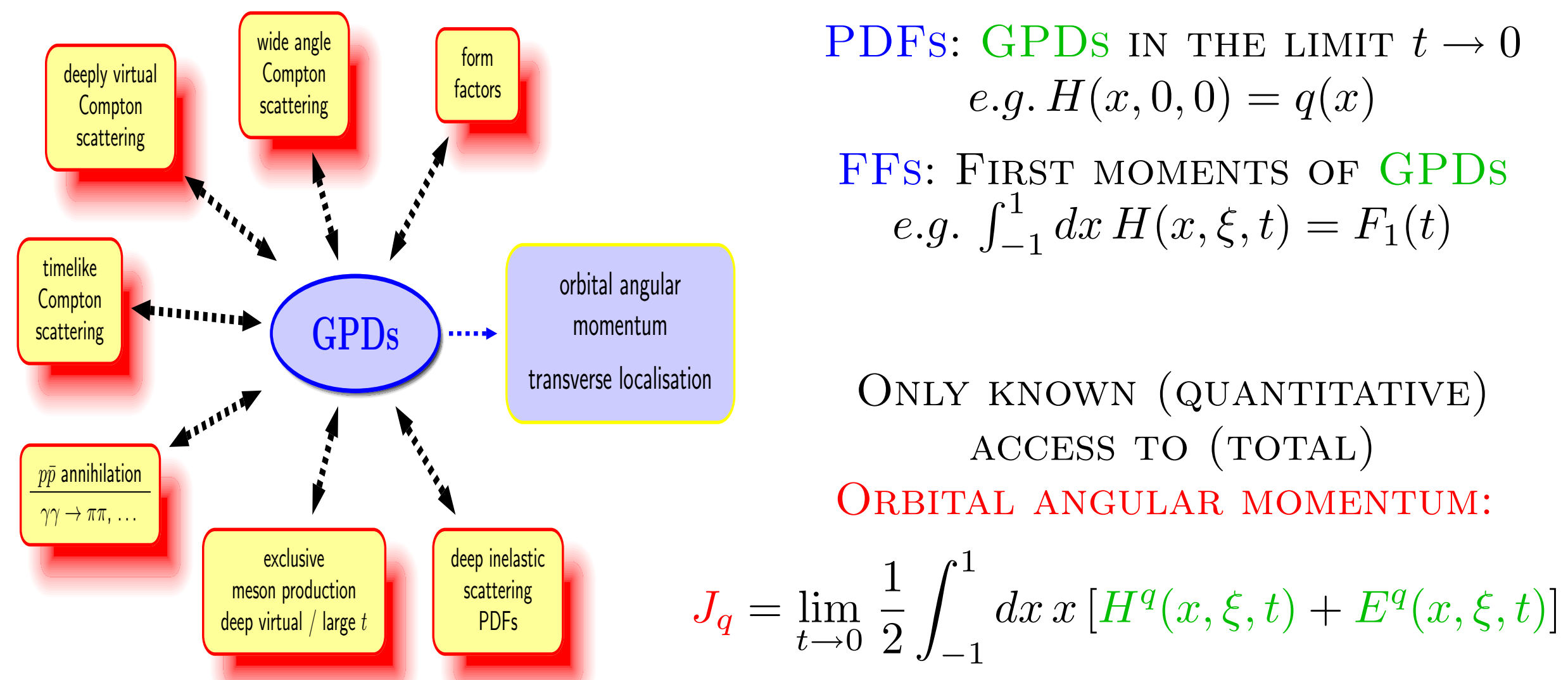

(ORiginal) HERMES Motivation:

Nucleon (Long.) Spin Structure: $1 / 2=\underbrace{1 / 2(\overbrace{\Delta u+\Delta d+\Delta s}^{\sim 30 \%})+\overbrace{L_{q}}^{?}}_{J_{q}=?}+\overbrace{J_{g}}^{?}$ 


\section{HowTo AcCess GPDs VIA DVCS?}

DVCS FinAl STATE $e+p \rightarrow e^{\prime}+p^{\prime}+\gamma$ IS Indistinguishable FROM ThE Bethe-Heitler Process $(\mathrm{BH}) \rightarrow$ Amplitudes add Coherently

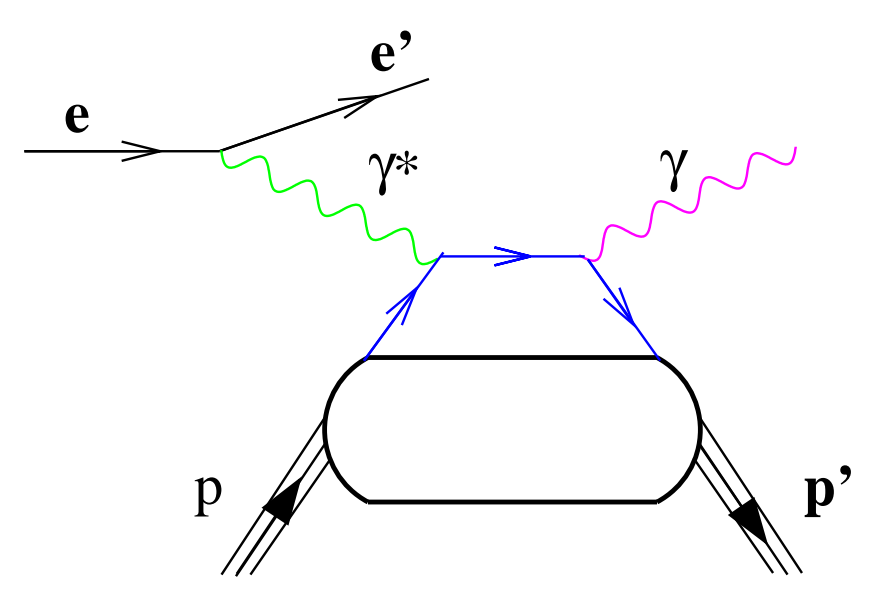

Fixed-TARget, Collider

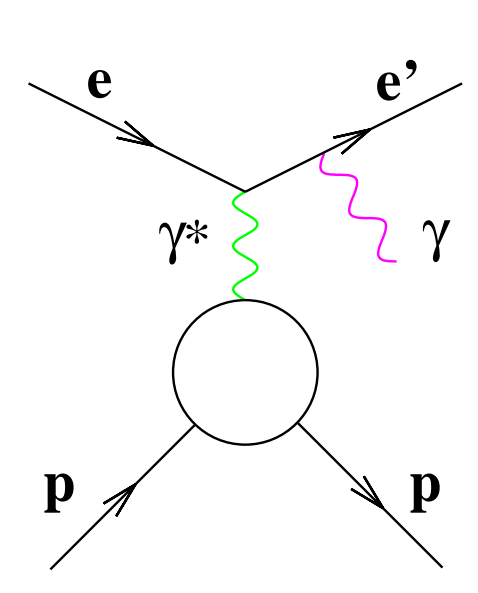

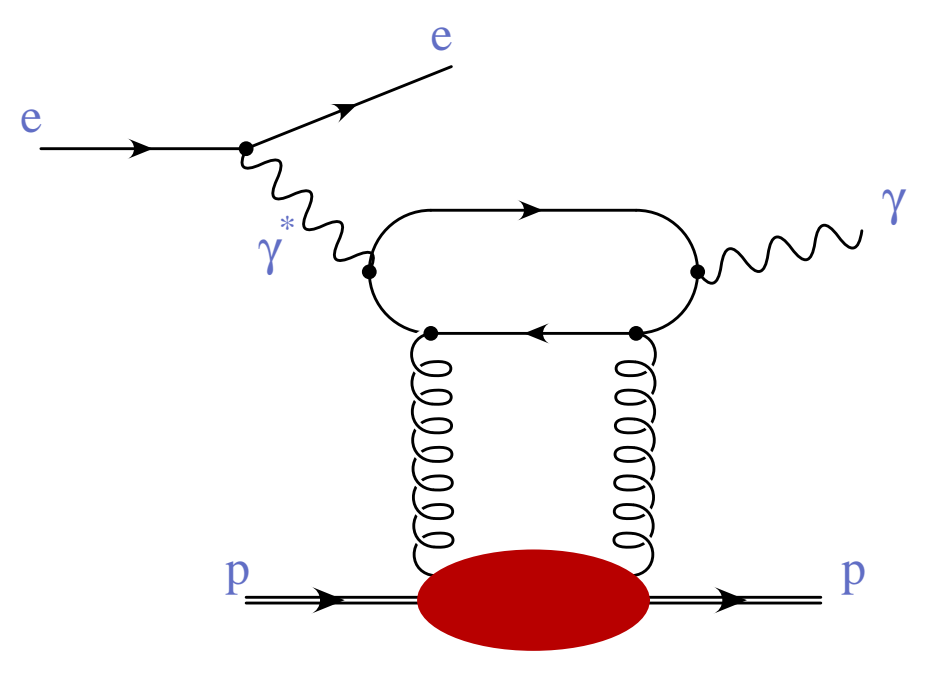

Collider

Photon-Production CROSS SECTION:

$$
d \sigma \propto\left|\tau_{\mathrm{DVCS}}+\tau_{\mathrm{BH}}\right|^{2}=\left|\tau_{\mathrm{DVCS}}\right|^{2}+\left|\tau_{\mathrm{BH}}\right|^{2}+\underbrace{\left(\tau_{\mathrm{DVCS}}^{*} \tau_{\mathrm{BH}}+\tau_{\mathrm{BH}}^{*} \tau_{\mathrm{DVCS}}\right)}_{I}
$$


$d \sigma \propto\left|\tau_{\mathrm{BH}}\right|^{2}+\underbrace{\left(\tau_{\mathrm{DVCS}}^{*} \tau_{\mathrm{BH}}+\tau_{\mathrm{BH}}^{*} \tau_{\mathrm{DVCS}}\right)}_{I}+\left|\tau_{\mathrm{DVCS}}\right|^{2}$

$\left|\tau_{\text {BH }}\right|^{2}$ CAlCulable in QED With The KNOWLEdGe OF The Form FaCtors

$I \propto \pm\left(c_{0}^{I}+\sum_{n=1}^{3} c_{n}^{I} \cos (n \phi)+\lambda \sum_{n=1}^{3} s_{n}^{I} \sin (n \phi)\right)$

DVCS CROSS SECTION (H1, ZeUS):

MEASUREMENT INTEGRATED OVER $\phi$

$\rightarrow I=0($ AT TWIST -2$)$, SUBTRACT $\left|\tau_{\mathrm{BH}}\right|^{2}$

(GPDS ENTER IN QUADRATIC COMBINATIONS)

AzIMUTHAL ASYMMETRIES

(HERMES, JLAB):

DVCS AMPLITUdES DIRECTLY ACCESSIBLE

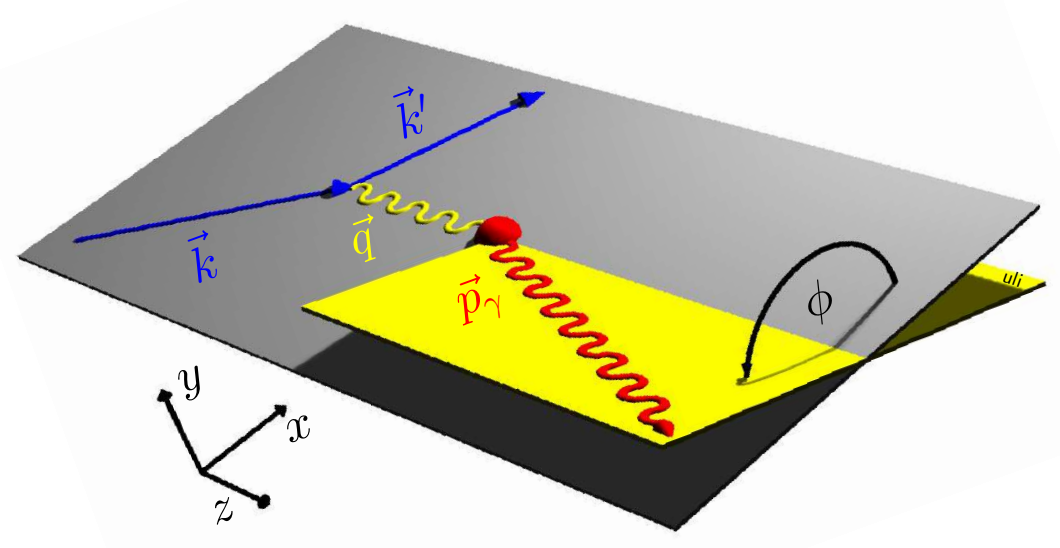

Via I $\Rightarrow$ Magnitude + Phase!!!

(GPDS ENTER IN LINEAR COMBINATIONS) 


\section{Azimuthal Asymmetries}

$$
I \propto \pm\left(c_{0}^{I}+\sum_{n}\left[c_{n}^{I} \cos (n \phi)+\lambda s_{n}^{I} \sin (n \phi)\right]\right)
$$

Beam-Spin Asymmetry (BSA) and Beam-Charge Asymmetry (BCA) ON UNPOLARIZED TARGET:

$$
\begin{aligned}
\mathrm{BSA}: & d \sigma\left(\overrightarrow{e^{+}} p\right)-d \sigma\left(\overleftarrow{e^{+}} p\right) \sim s_{1, u n p}^{I} \sin (\phi) \sim \sin (\phi) \times \operatorname{Im} M_{u n p}^{1,1} \\
\mathrm{BCA}: & d \sigma\left(e^{+} p\right)-d \sigma\left(e^{-} p\right) \sim c_{1, u n p}^{I} \cos (\phi) \sim \cos (\phi) \times \operatorname{Re} M_{u n p}^{1,1}
\end{aligned}
$$

(Higher Twist/Order $\rightarrow \cos 2 \phi, \cos 3 \phi, \sin 2 \phi$ )

$M_{u n p}^{1,1}=F_{1}(t) H_{1}(\xi, t)+\frac{x_{B}}{2-x_{B}}\left(F_{1}(t)+F_{2}(t)\right) \widetilde{H}_{1}(\xi, t)-\frac{t}{4 M^{2}} F_{2}(t) E_{1}(\xi, t)$

$\left\langle x_{B}\right\rangle,\langle-t\rangle \approx 0.1 \Rightarrow$ COMPTON Form-FaCTOR $H_{1} \Rightarrow$ GPD $H$

NEED BOTH CHARGES AND POLARIZED BEAM

$$
\Rightarrow \text { HERA!!! }
$$




\section{The HERA ACCELERATOR AT DESY (HAMBURG)}

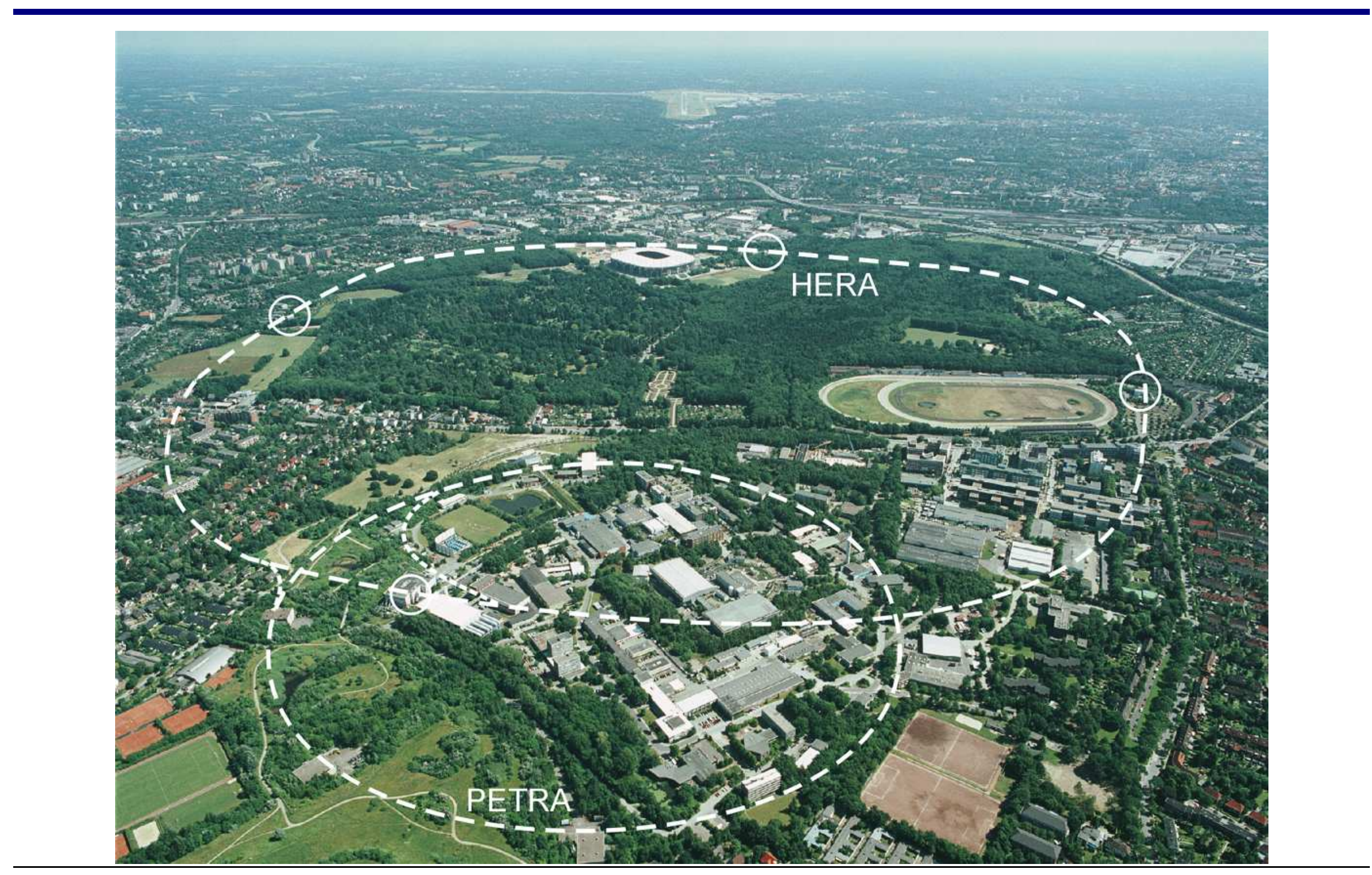

Frank Ellinghaus, Puerto Rico, May 2006 
HERA BEAM: 27.6 GEV, $e^{+}$AND $e^{-},\langle P\rangle \approx 35-55 \%$

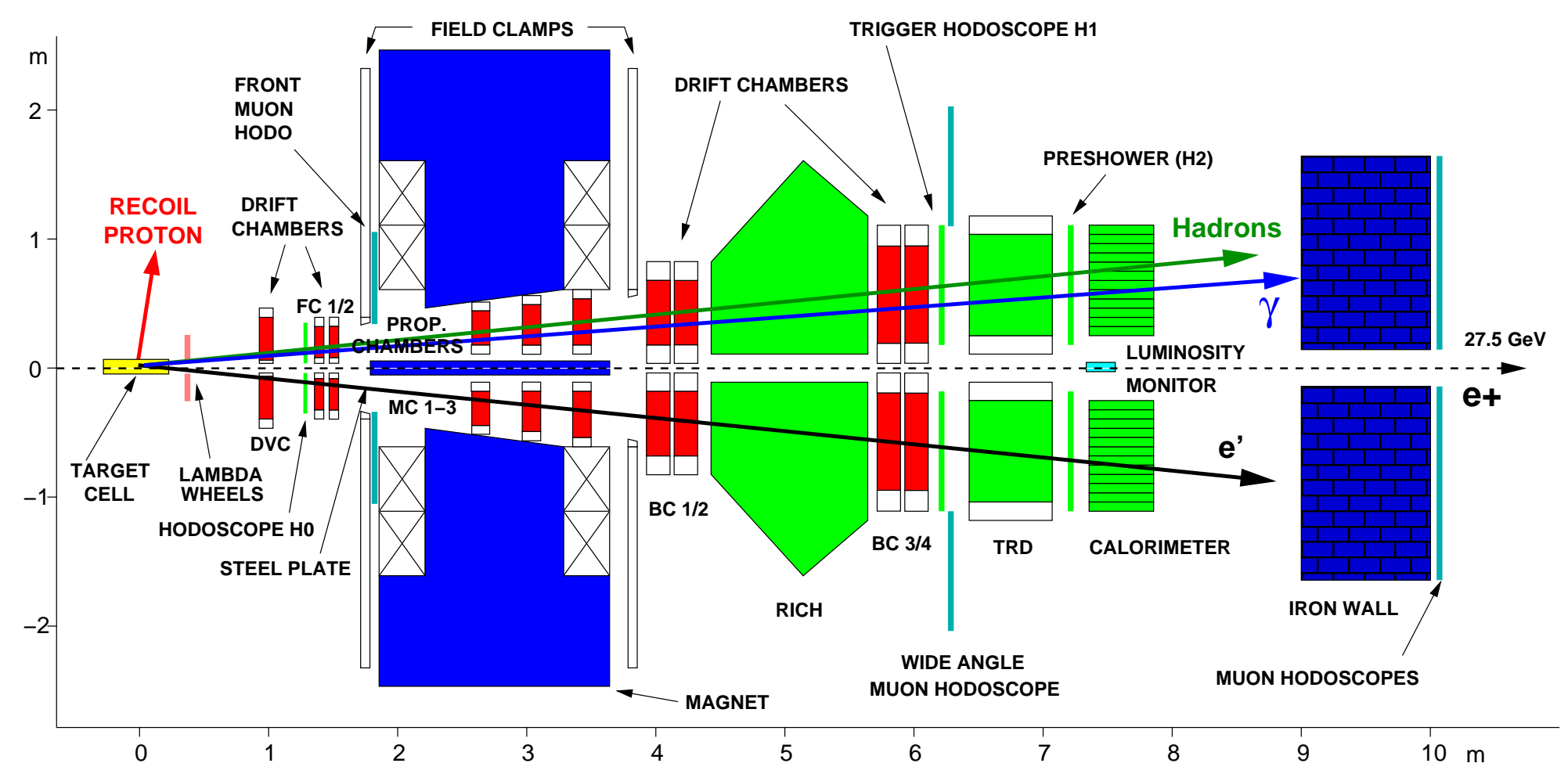

Events With EXACTLY ONE DIS-POSITRON/DIS-ELECTRON AND EXACTLY ONE PHOTON IN THE CALORIMETER (OR ONE $\left(\rho^{0} \rightarrow\right) \pi^{+} \pi^{-}$PAIR )

No RECOIL DETECTION (YET) $\Rightarrow$ ExCLusivity Via ... 
$M_{x}^{2} \equiv\left(q+p-p_{\gamma}\right)^{2} \Rightarrow \mathrm{MC}$ FOR BACKGROUND AND CUTS $(\rightarrow$ RESOLUTION $) !$

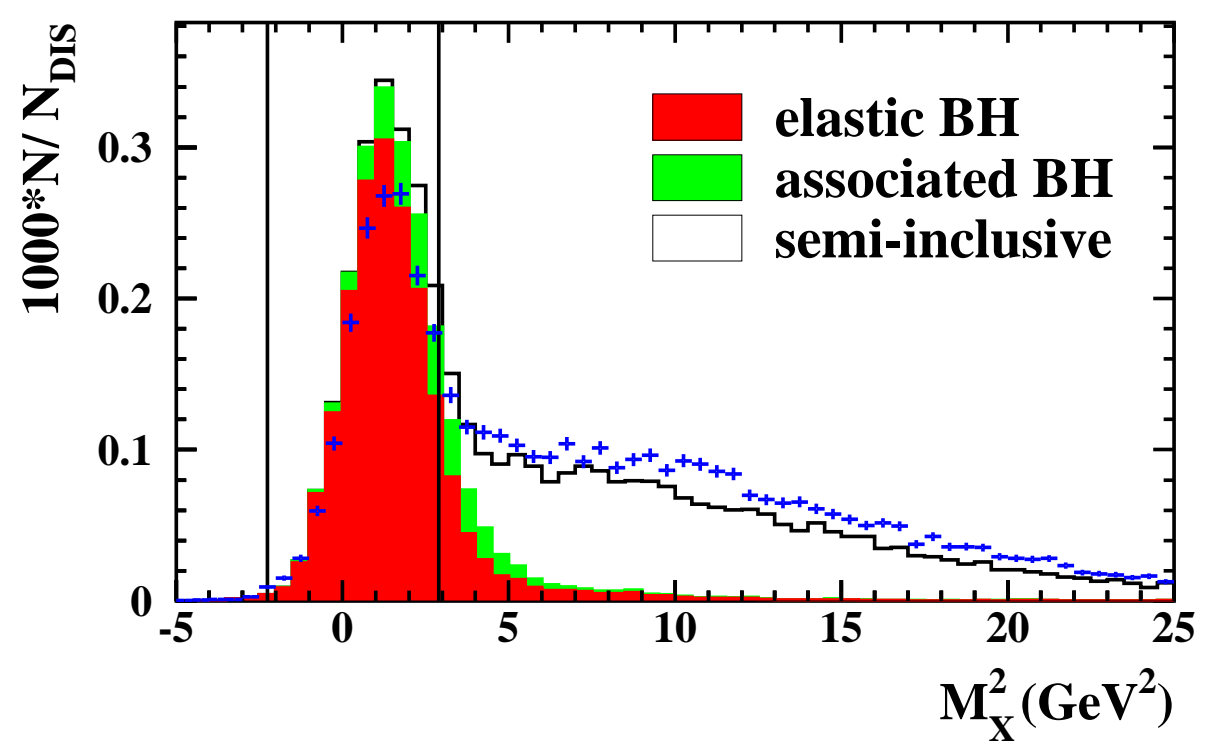

- ELASTIC BH $\left(e p \rightarrow e^{\prime} p^{\prime} \gamma\right)$

- ASSOCIATED BH (MAINLY $e p \rightarrow e^{\prime} \Delta^{+} \gamma$ )

- SEMI-INCLUSIVE (MAINLY $\left.e p \rightarrow e^{\prime} \pi^{0} X\right)$

- EXCLUSIVE $\pi^{0}\left(e p \rightarrow e^{\prime} \pi^{0}\right)$ NOT SHOWN (SMALL)

Not Simulated: DVCS PROCESS (DVCS C.S. "UNKNOWN", DVCS $<<$ BH) + Radiative CORRECTIONS TO BH $(\rightarrow$ EXCL. PEAK OVERESTimated, BG UNDERESTIMATED)

$\Rightarrow$ "ExClusive" BIN $\left(-1.5<M_{x}<1.7 \mathrm{GeV}\right)$

$\Rightarrow$ Overall BaCkground CONTRIBUtion $\approx 15 \%$ 


\section{BeAm-Spin Asymmetry (BSA)}

$$
A_{\mathrm{LU}}(\phi)=\frac{1}{<\left|P_{b}\right|>} \frac{\vec{N}(\phi)-\overleftarrow{N}(\phi)}{\vec{N}(\phi)+\overleftarrow{N}(\phi)}
$$

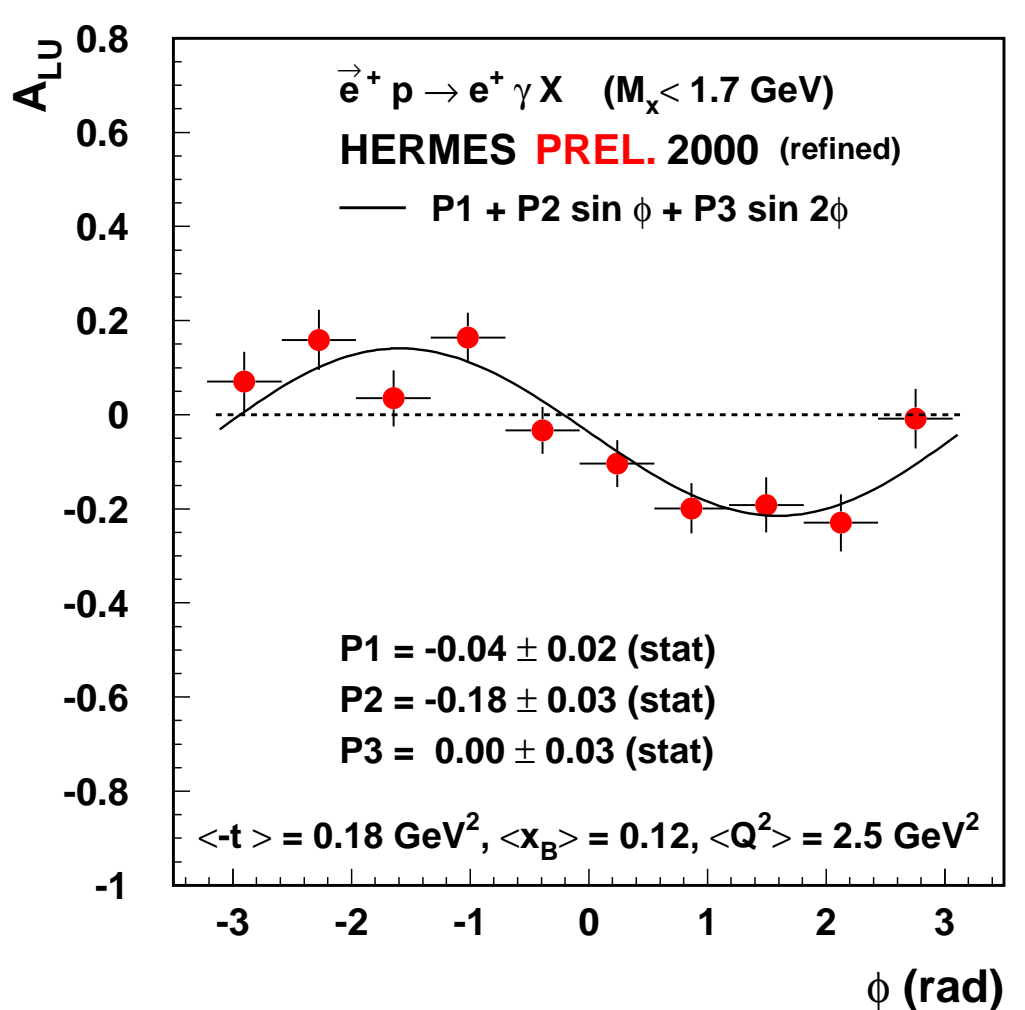

$A_{\mathrm{LU}}$ IN EXCLUSIVE BIN: ExpECTED $\sin (\phi)$ DEPENDENCE $\Rightarrow \operatorname{Im} M_{u n p}^{1,1}$

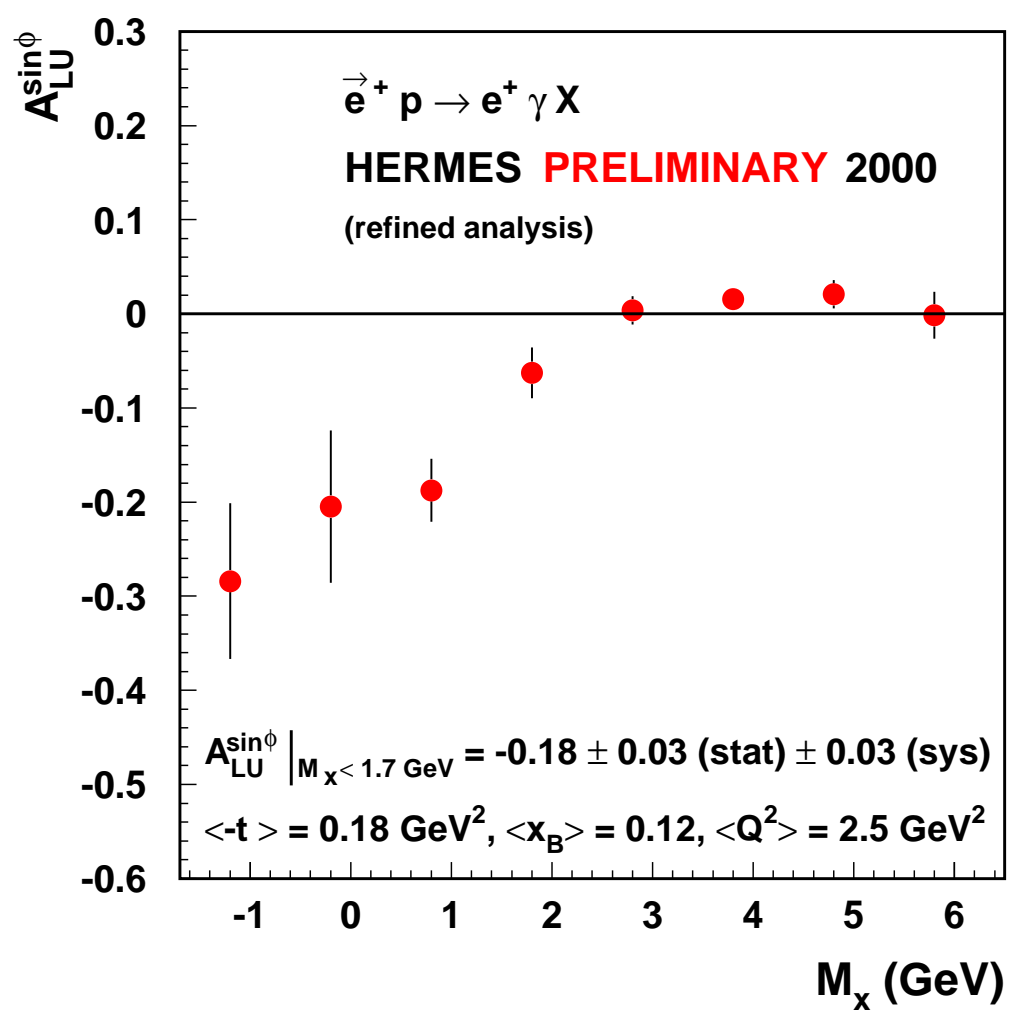

$\sin (\phi)-$ MOMENT IN NON-EXCLUSIVE REGION: SMALL AND SLIGHTLY POSITIVE $\left(\rightarrow \pi^{0}\right)$

$\underline{(\text { RESUltS FROM 1996/97 } \rightarrow \text { PRL 87, } 182001 \text { (2001)) }}$

Frank Ellinghaus, Puerto Rico, May 2006 
BCA: BeAM-ChARGE Asymmetry (hep-ex/0605108, subm. to PRL)

$A_{\mathrm{C}}(\phi)=\frac{N^{+}(\phi)-N^{-}(\phi)}{N^{+}(\phi)+N^{-}(\phi)} \propto I \propto \pm\left(c_{0}^{I}+\sum_{n=1}^{3} c_{n}^{I} \cos (n \phi)+\lambda \sum_{n=1}^{2} s_{n}^{I} \sin (n \phi)\right)$

$\Rightarrow$ Calculate "Symmetrized" BCA $(\phi \rightarrow|\phi|)$ To GeT Rid OF All $\sin (\phi)-$ DEPENDENCES DUE TO POLARIZED BEAM.

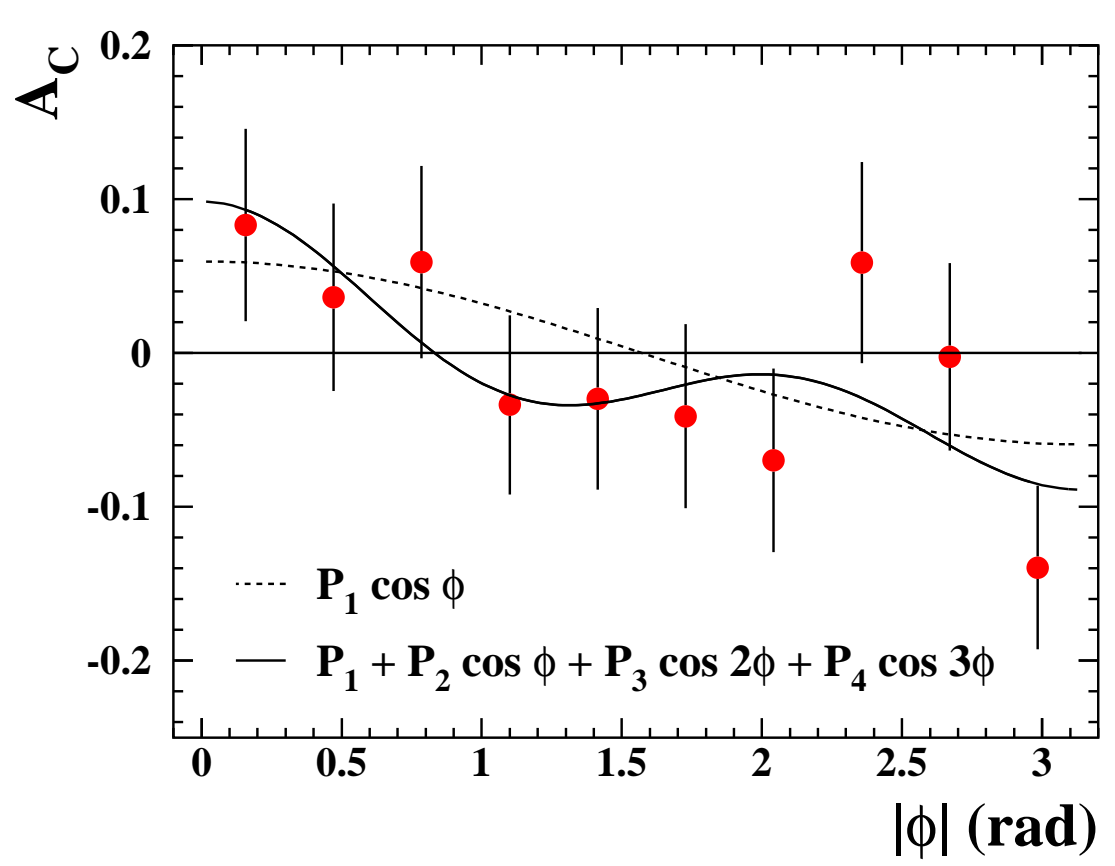

$A_{\mathrm{C}}$ In EXClusive Bin: ExpeCted $\cos (\phi)$ DEPENDENCE $\Rightarrow \operatorname{Re} M_{u n p}^{1,1}$

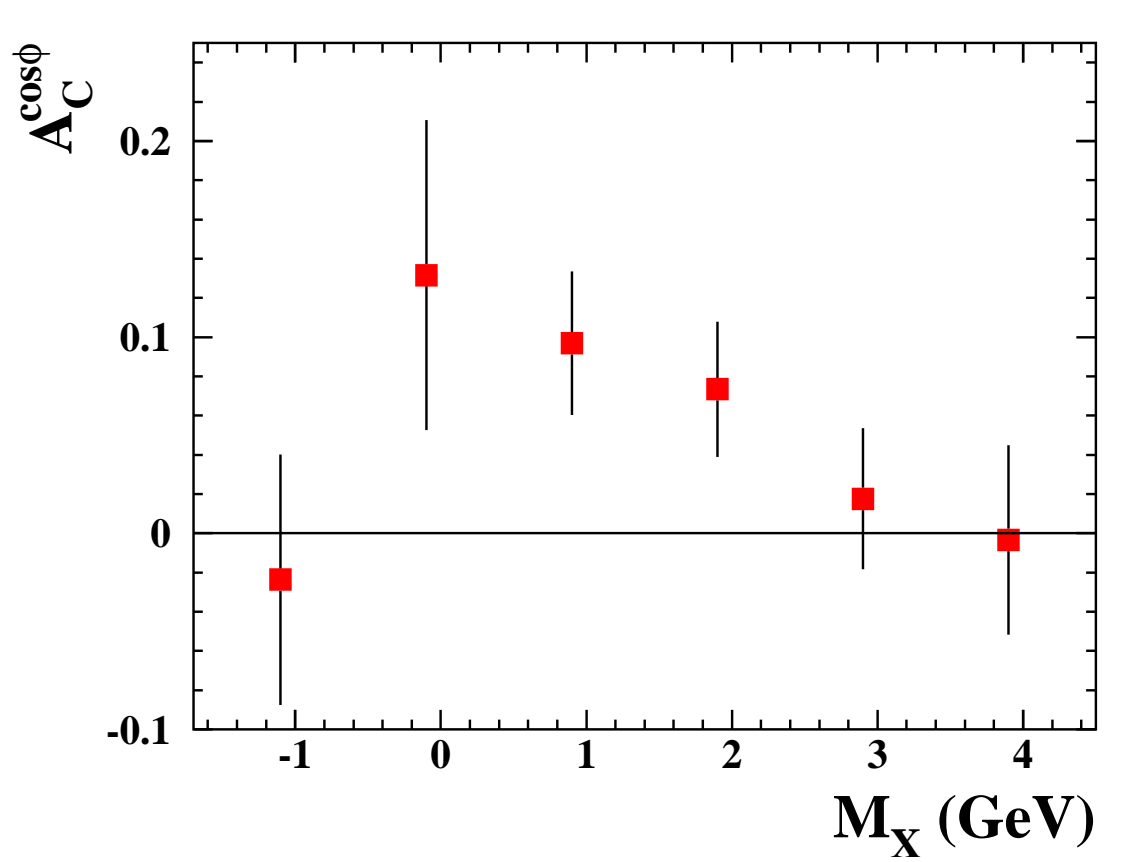

$\cos (\phi)$-Moments Zero AT Higher MISSING MASS 


\section{Beam-Charge Asymmetry (BCA) versus $-t$}

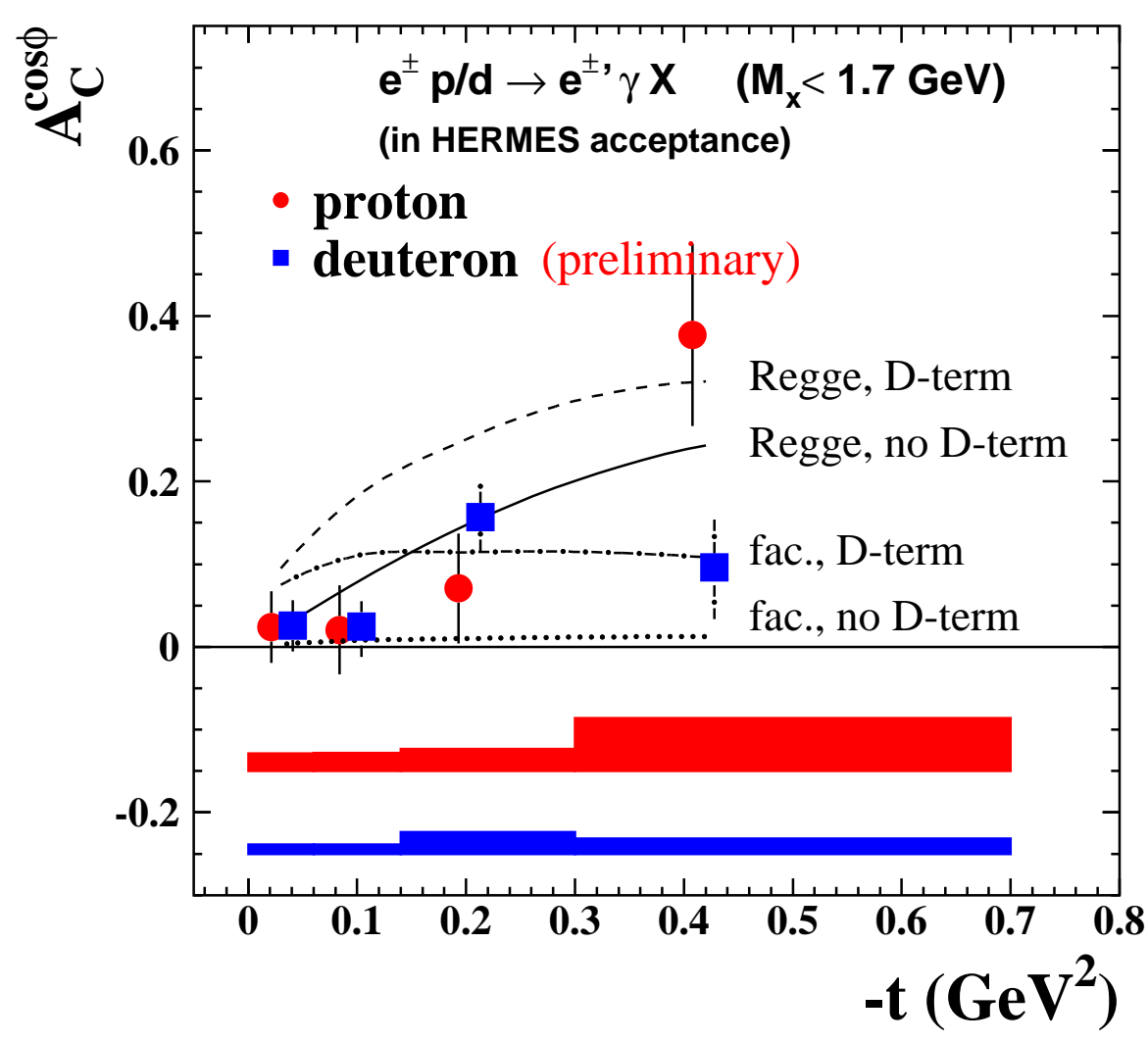

Also: DVCS ON DEUTERIUM: COHERENT PRODUCTION ON D ONLY IN FIRST $t$-BIN $(\approx 40 \%)$

$\Rightarrow$ NO EFFECT SEEN

$\rightarrow \approx$ P-TARGET

Possible DifFERENCE IN LAST BIN $(\rightarrow$ NEUTRON $)$

GPD Model Calc. at aVerage Kinematic Values Per Bin (COde by Vanderhaeghen, Guichon, Guidal)

TINY $e^{-} p$ SAMPle $\left(L \approx 10 \mathrm{~PB}^{-1}\right) \Rightarrow$ REGGE + D-TERM DisfaVorED $\Rightarrow t$-DEPENDENCE OF BCA HAS HIGH SENSITIVITY TO GPD MODELS! 


\section{MORE ON H TO COME}

Recoil Detector AND UNPOL. TARgets (2006/2007)

- ENSURES EXCLUSIVITY OF EVENTS

- Semi-InClusive BACKGROUND $5 \% \Rightarrow \ll 1 \%$

- Associated BACKGROUND 10\% $\Rightarrow \approx 1 \%$
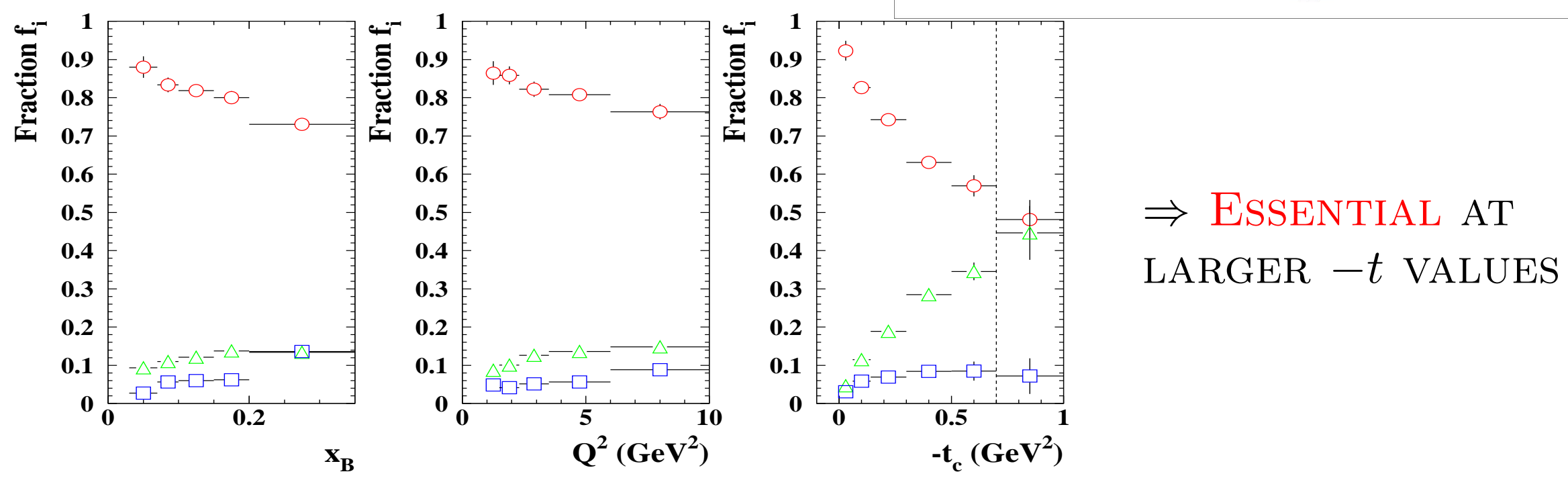

Frank Ellinghaus, Puerto Rico, May 2006 


\section{$\mathrm{BCA}$}

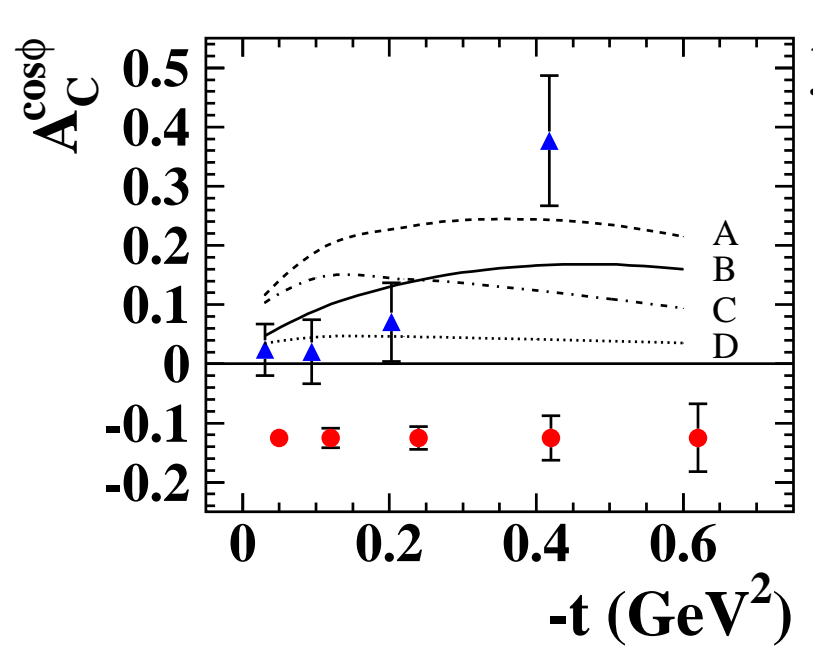

BSA

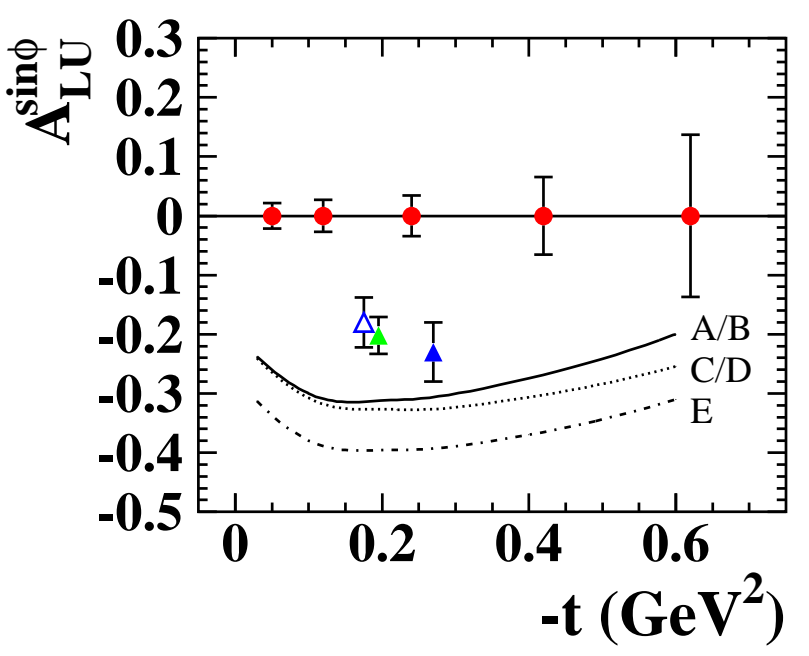

$\triangle$ : HERMES PRELIM./PUBLISHED

$\triangle$ : CLAS, PRL, $2001(x-1)$

•: $1 \mathrm{fb}^{-1} e^{+}$AND $0.25 \mathrm{fb}^{-1} e^{-}$, POL. $=35 \% \quad$ (EXP. 2002-2007 DATA)

BCA: High SENSITIVITy TO $t$-DEPENDENCE (FACT./REGGE) AND D-TERM BSA: HIGHEST SENSITIVITY TO $b_{s}$ PARAMETER IN PROFILE FUNCTION

Possibility TO "MAP ouT" GPD $H^{u}$ IN THE FinAl TWO HERA YEARs. 


\section{What about the GDP $E$ ?}

REMEMBER:

$$
J_{q}=\lim _{t \rightarrow 0} \frac{1}{2} \int_{-1}^{1} d x x\left[H^{q}(x, \xi, t)+E^{q}(x, \xi, t)\right]
$$

GPD $E$ IS ALWAYS KINEMATICALLY SUPPRESSED, EXCEPT IN: $A_{U T}$ : UNPOLARIZED BEAM, TRANSVERSELY POL. TARGET

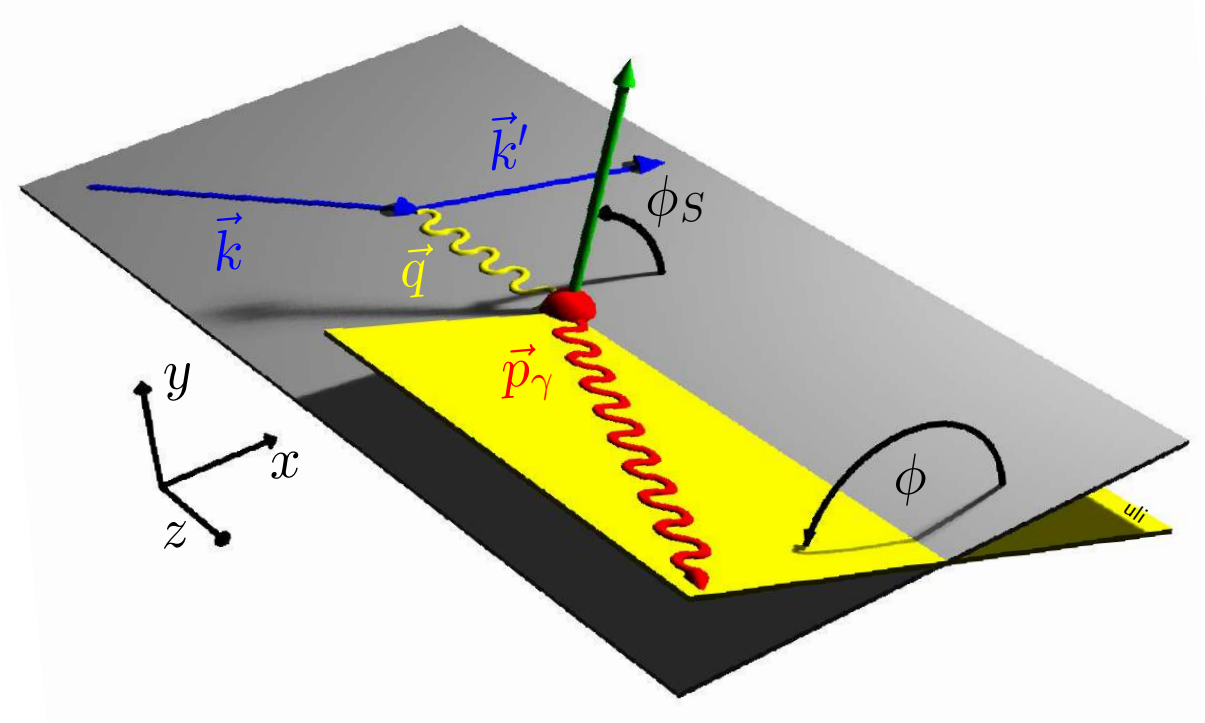

$A_{U T}\left(\phi, \phi_{s}\right)=\frac{1}{\left|P_{T}\right|} \cdot \frac{d \sigma^{\Uparrow}\left(\phi, \phi_{s}\right)-d \sigma^{\Downarrow}\left(\phi, \phi_{s}^{\prime}\right)}{d \sigma^{\Uparrow}\left(\phi, \phi_{s}\right)+d \sigma^{\Downarrow}\left(\phi, \phi_{s}^{\prime}\right)}$

$\propto \operatorname{Im}\left[F_{2} \mathcal{H}-F_{1} \mathcal{E}\right] \cdot \sin \left(\phi-\phi_{S}\right) \cos \phi+\operatorname{Im}\left[F_{2} \widetilde{\mathcal{H}}-F_{1} \xi \widetilde{\mathcal{E}}\right] \cdot \cos \left(\phi-\phi_{S}\right) \sin \phi$ 
DATA TAKING WITH TRANSVERSE HYDROGEN TARGET FINISHED $\approx 10$ MiLlion ON TAPE, HALF THE DATA (2002-2004) ANALYZED

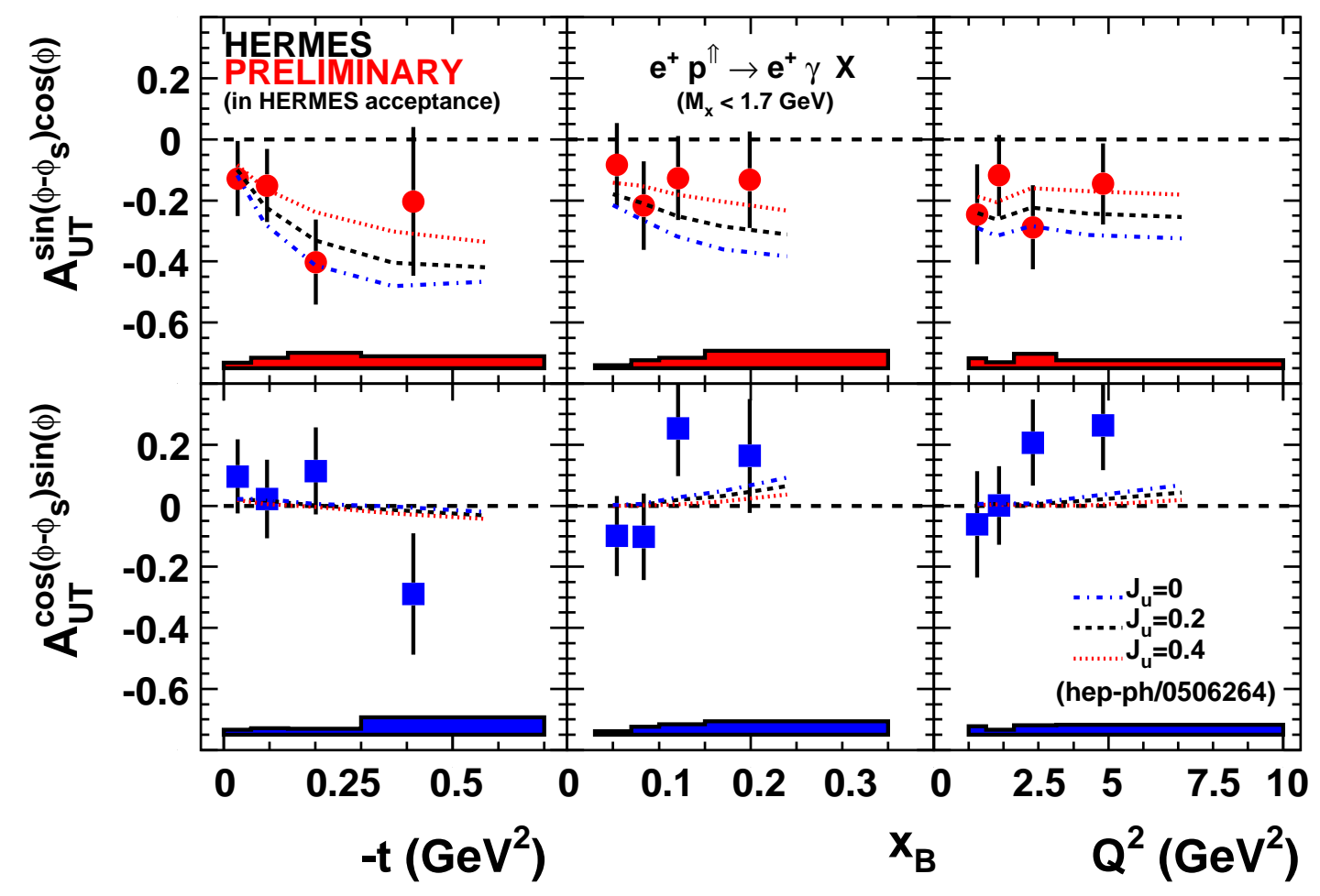

$A_{U T}^{\sin \left(\phi-\phi_{s}\right) \cos \phi}$ LARGELY INDEPENDENT ON ALL MODEL PARAMETERS BUT $J_{u}$ (F.E., NowAK, Vinnikov, Ye, HeP-PH/0506264)

$\Rightarrow$ First Model DePEndent extraction of $J_{u}$ POSsible! 


\section{First Constraint on Angular Momentum !}

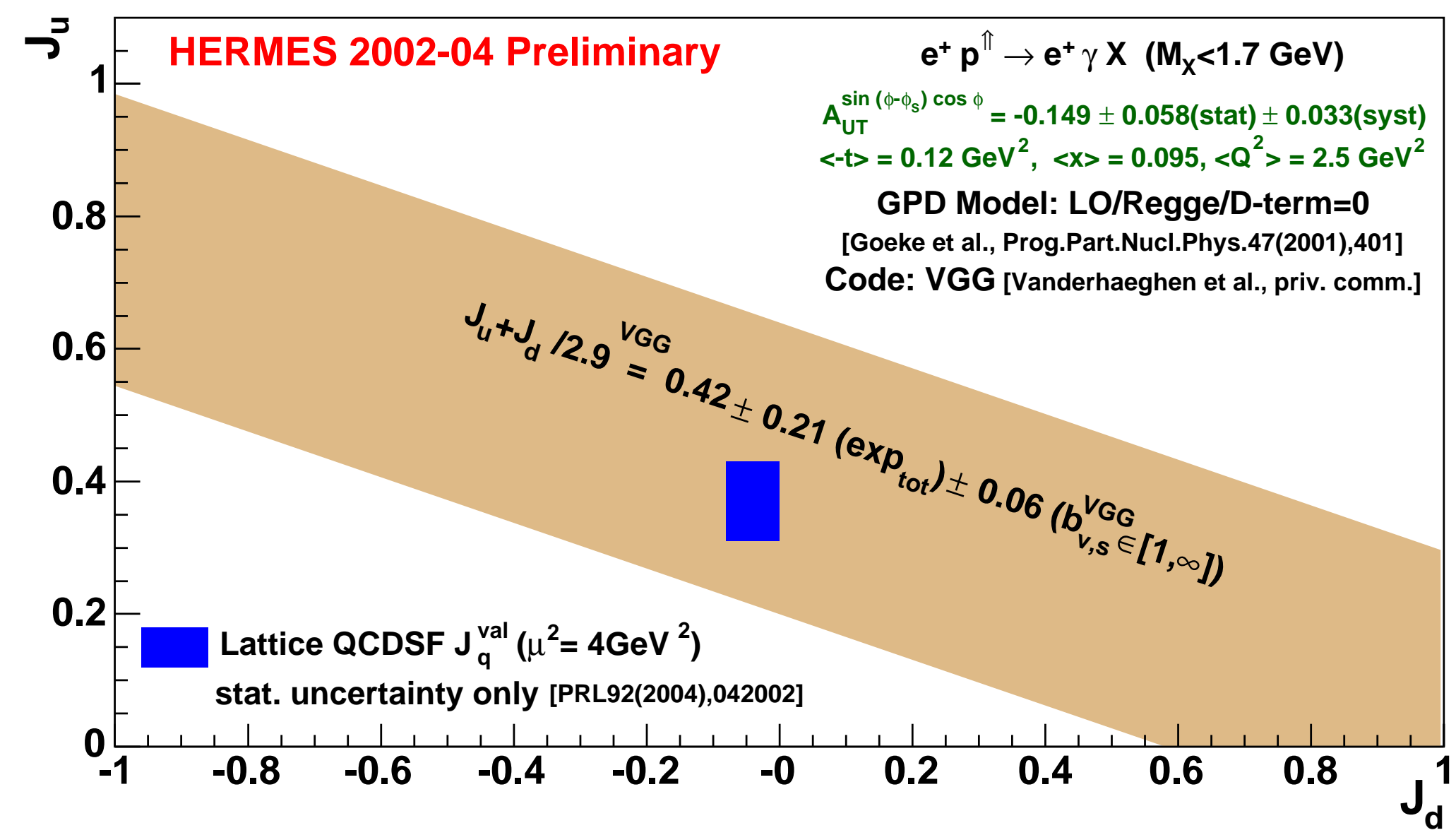

$\Rightarrow$ First MODEL DEPENDENT CONSTRAINT ON TOTAL QUARK ANGULAR MOMENTUM $J_{u}, J_{d}$. 


\section{Exclusive Vector Meson Production}

THE (ONLY) OTHER (PROMISING)

ACCESS TO E $(\mathrm{J})$ (ON A P TARGET): $A_{U T}$ in ExClusive $\rho^{0}$ PROduCtion:

$$
e p \rightarrow e p \rho^{0}
$$

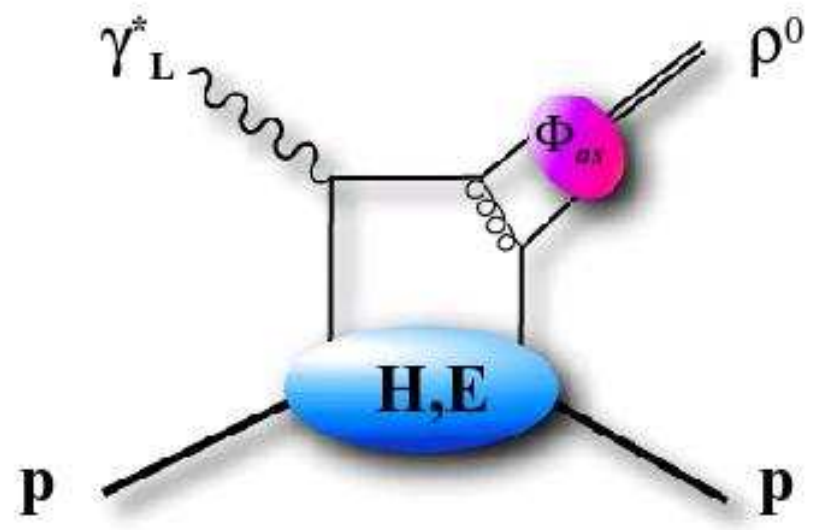

EVENT SELECTION:

- $\rho^{0} \rightarrow \pi^{+} \pi^{-}$,

- No RECOIL DETECTION

- $\rightarrow$ MISSING ENERGY
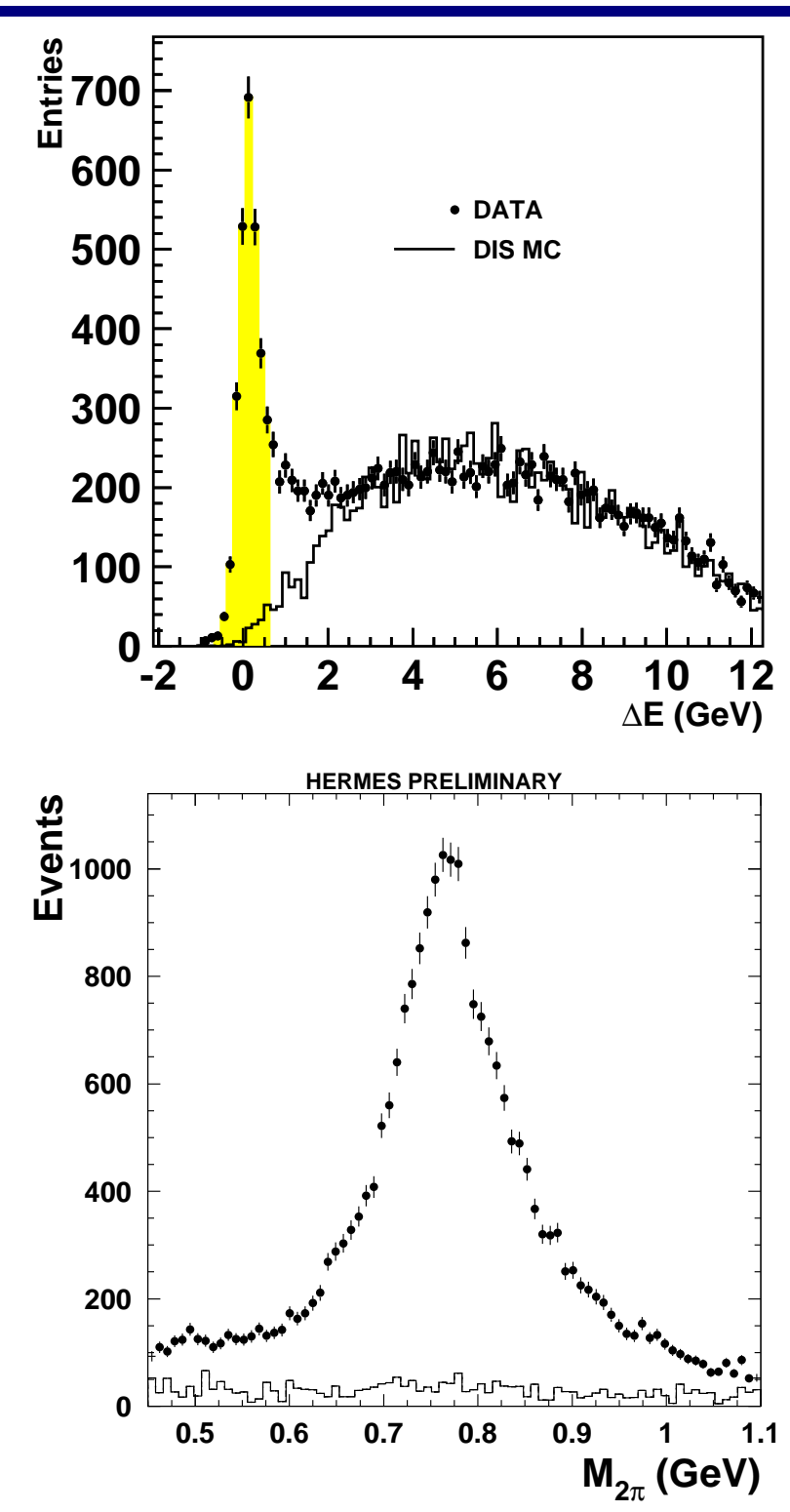


$$
A_{U T}\left(\phi, \phi_{s}\right)=\frac{1}{\left|P_{T}\right|} \cdot \frac{d \sigma^{\Uparrow}\left(\phi, \phi_{s}\right)-d \sigma^{\Downarrow}\left(\phi, \phi_{s}\right)}{d \sigma^{\Uparrow}\left(\phi, \phi_{s}\right)+d \sigma^{\Downarrow}\left(\phi, \phi_{s}\right)} \propto H E \cdot \sin \left(\phi-\phi_{S}\right)
$$

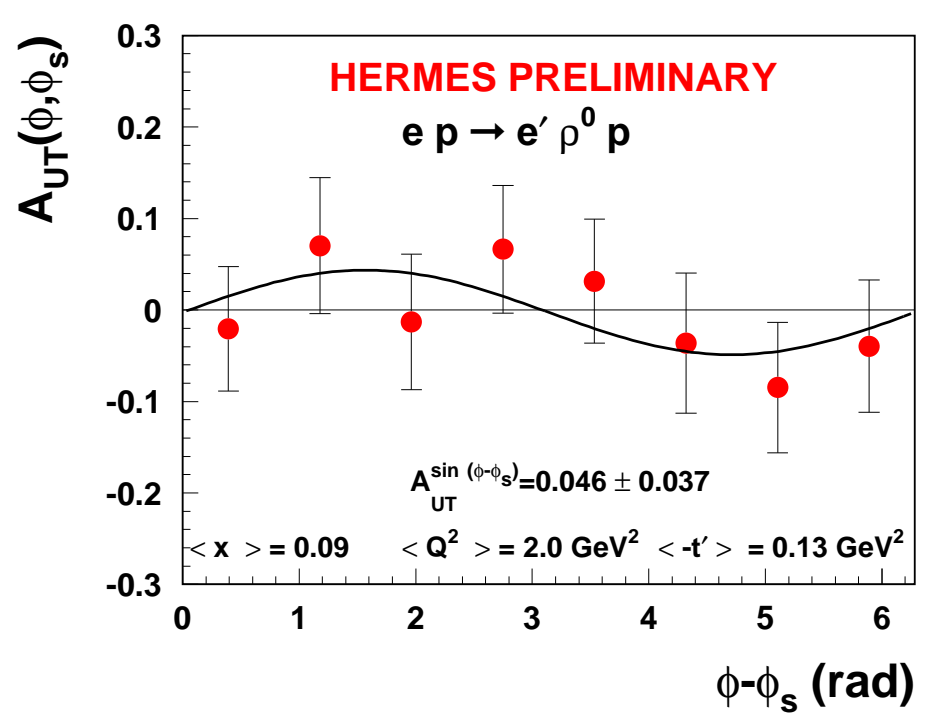

EXPECTED sin $\phi$ BEHAVIOR (NO L/T SEPARATION YET)

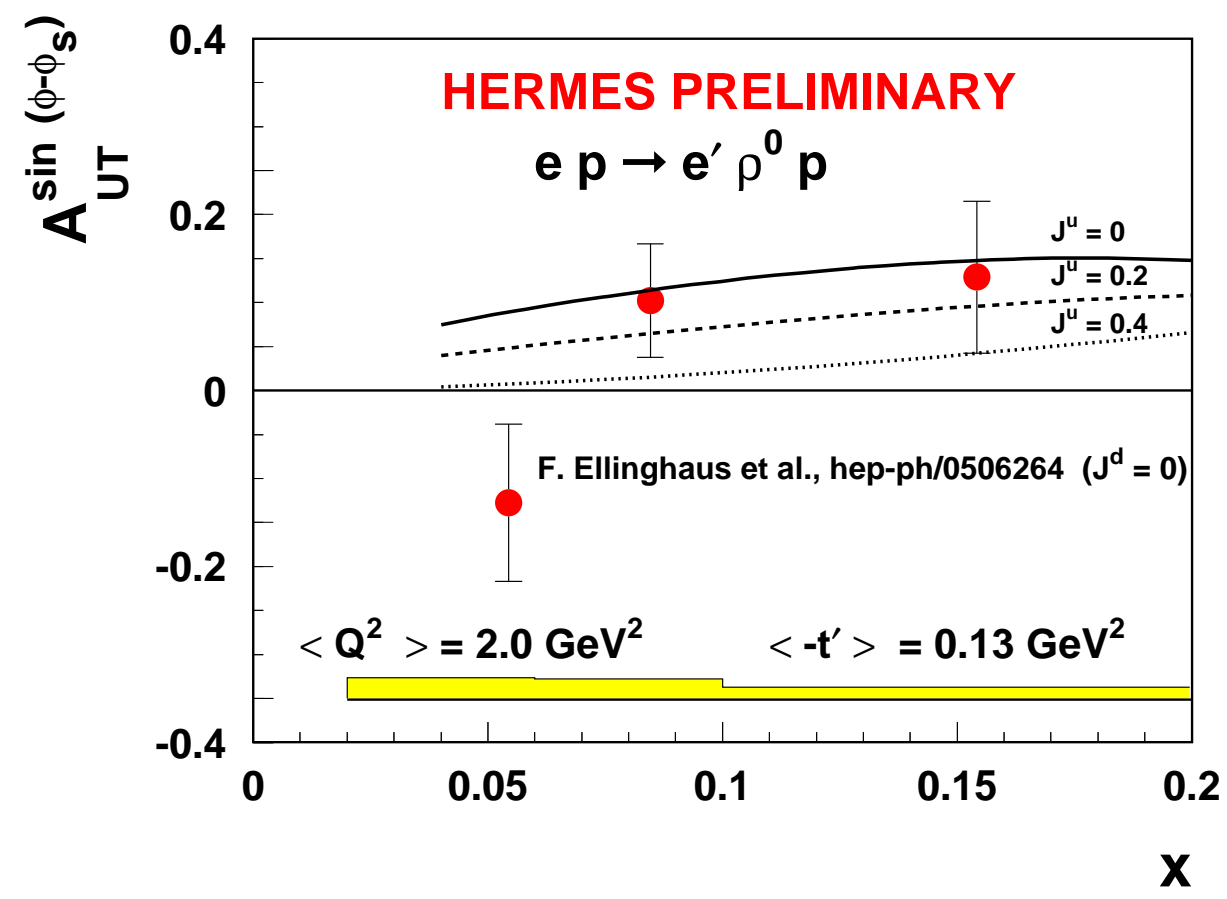

Agreement With theoretical Calculation.

(CALCULATION/FACTORIZATION PROOF FOR LONGITUDINAL PHOTONS ONLY)

AGAin: SAME Size DATA SET to COME, $A_{U T}^{\rho}$ Less SENSitive to $J_{u}$ When COMPARED TO $A_{U T}^{D V C S} \rightarrow$ PROVIDE ADDITIONAL CONSTRAINTS 


\section{SUMMARY}

- Hard exclusive Processes probe GPDS

- Results so far in General agreement With BASIC MODEls AND ASSUMPTIONS.

- 2006/2007 Data taking (+Recoil Detector) Devoted to Exclusive REACTIONS:

$\rightarrow$ "MAP OUT" GPD $H^{u}$ VIA DVCS BEAM-SPIN AND BEAM-Charge ASYMMETRY

- DVCS ON TRANSVERSE POLARIZED TARGET:

First model DePEndent CONSTRAint on the total ANGUlar MOMENTUM OF U-QUARKS $\left(J_{u}\right)$ AND D-QUARKS $\left(J_{d}\right)$ IN THE NUCLEON. 